This supplement also contains articles that describe the broad experience with terazosin in addressing issues in hypertension. The pharmacokinetics of this agent are described as well as its clinical applications. Its efficacy has been examined carefully in differing racial groups, and considerable data have also been accumulated on its safety profile. Terazosin has been found to be effective in both combination therapy and monotherapy for the treatment of hypertension, and these experiences are described in this supplement.

In summary, these proceedings comprise a series of articles that address the modern concept of hypertension and provide a broad basis for understanding and treating this very interesting condition. $\mathrm{Al}$ though decreasing blood pressure is a pivotal part of treating hypertensive patients, other issues must also be considered. The selective $\alpha_{1}$-adrenergic-blocking agent terazosin is now being examined in this context, and these reports should help to position this agent as an important part of the armamentarium to be used in achieving our treatment goal of protecting hypertensive patients from major cardiovascular events.

\title{
Clinical implications of pathophysiologic changes in the midlife hypertensive patient
}

\begin{abstract}
Both aging and hypertension decrease cardiac output through a lower stroke volume and a diminished $\beta$-adrenergic responsiveness. In parallel, the vascular resistance increases because of vascular hypertrophy. In addition, in hypertension the $\alpha$-adrenergic responsiveness is enhanced. Aging and hypertension are also associated with an increase in plasma cholesterol and insulin values. These alterations in cholesterol and insulin levels become particularly pronounced in middle-aged patients with hypertension. $\alpha$-Adrenergic-blocking agents have a positive effect on lipids and insulin resistance. The shift toward enhanced $\alpha$-adrenergic responsiveness, and the fact that vasodilators do not diminish exercise performance favor the use of $\alpha$-blockers as a first drug in middle-aged patients with hypertension. Blood pressure lowering still remains the paramount goal, and these compounds should be used only if proved to be efficacious in a given individual patlent. (AM HEART J 1991;122:886-91.)
\end{abstract}

Stevo Julius, MD, ScD Ann Arbor, Mich.

Both hypertension and aging elicit similar cardiovascular changes. The difference is in the time course: slower with aging, faster with hypertension. The pace of cardiovascular changes is particularly accelerated when both conditions coexist. It is important to understand that aging and hypertension are dynamic processes. Consequently, determining which patho-

From the Division of Hypertension, Department of Internal Medicine, Uni versity of Michigan Medical Center.

Reprint requests: Stevo Julius, MD, ScD, Division of Hypertension, Department of Internal Medicine, University of Michigan Medical Center, 3918 Taubman Center, Ann Arbor, MI 48109-0356.

1/0/30199 physiologic changes are present in an individual patient may affect clinical decisions.

It is not difficult to visualize why hypertension and aging affect the circulation in a similar fashion; the overall wear and tear on the vessels and the heart might be similar if they were exposed to lower blood pressure over a longer period or to a higher pressure over a shorter period of time. However, the similarity between hypertension and aging extends beyond their immediate effects on circulatory organs. Both conditions are associated with hyperlipidemia and insulin resistance. The nature of this association is not clear, but in practical terms the frequent presence of metabolic abnormalities in middle-aged hypertensive subjects is clinically important. This arti- 


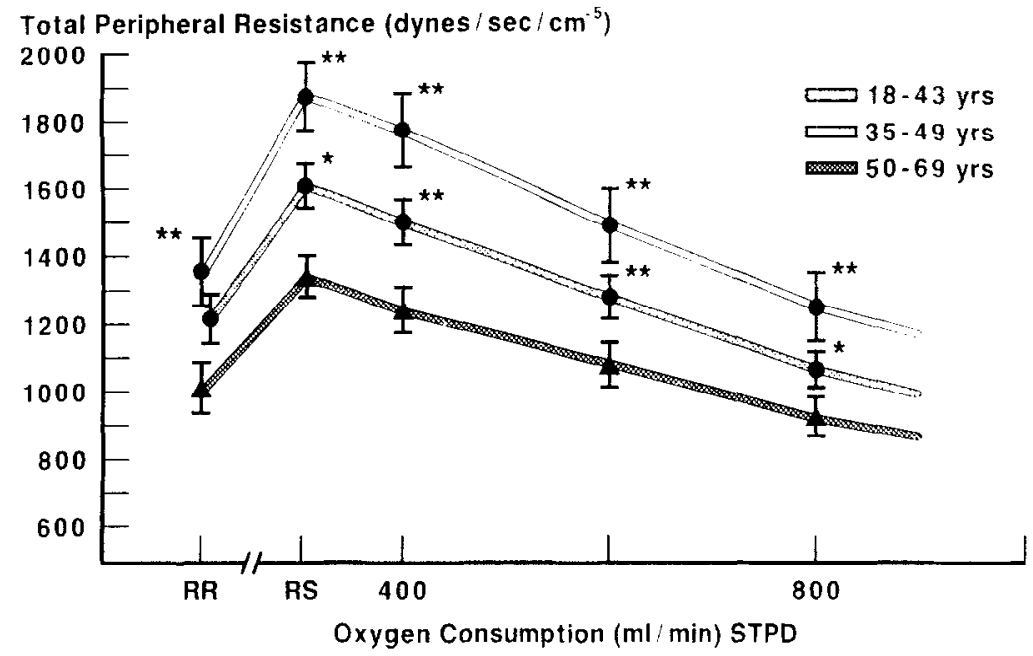

Fig. 1. Effect of exercise on the calculated total peripheral resistance in three age groups. The oxygen consumption is given at STPD conditions (standard temperature, pressure, dry). (Modified from Julius S, Amery A, Whitlock LS, Conway J. Influence of age on the hemodynamic response to exercise. Circulation 1967;36:222-30, by permission of The American Heart Association, Inc.)

cle describes the age- and blood pressure-dependent changes in cardiovascular function and how they may affect the choice of antihypertensive agents. A case will be made for the use of $\alpha$-blocking agents in middle-aged patients with hypertension.

\section{CHANGES IN STROKE VOLUME AND VASCULAR RESISTANCE}

Assessment by proper invasive hernodynamic methods shows that the cardiac output and stroke volume decrease with age. ${ }^{1-3}$ One study that found a normal cardiac output and an increased stroke volume in elderly subjects used radionuclide measurements. ${ }^{4}$ The authors claim that this aberrant finding reflects their selection of particularly healthy study subjects. However, radionuclide measurements of cardiac output in elderly subjects are not reliable.

Decreased cardiac output in elderly persons is frequently associated with an age-dependent blood pressure elevation. The hemodynamic end result of these trends in blood pressure and cardiac output is an increase in systemic vascular resistance with aging. Despite the intense stimulus to vasodilate, elderly subjects continue to show increased vascular resistance during all levels of exercise. ${ }^{1}$ This finding suggests that the increased resistance in elderly persons is caused by structural changes in the vessels. If the increased vascular resistance at rest were just a reflex response to the lower cardiac output, strong vasodilation during exercise should override the resting vasoconstriction.

In subjects of similar age, the stroke volume and cardiac output decrease and vascular resistance in- creases with increasing blood pressure levels. ${ }^{5}$ These effects of blood pressure are identical to the effects of aging. Studies in hypertension, however, have shed some light on the mechanism of these changes. The decrease in stroke volume appears very early in hypertension. During exercise, patients with hypertension respond like elderly subjects: at each level of exercise the vascular resistance progressively decreases but remains relatively elevated compared with that of control subjects ${ }^{6}$ (Fig. 1). Despite this progressive fall in resistance, stroke volume remains lower than in control subjects and reaches a plateau at lower exercise loads. ${ }^{7}$ If the higher afterload caused by higher resistance is the reason for the decreased stroke volume in hypertension, then during exercise as the resistance progressively decreases, the stroke volume should eventually become comparable with that observed in normotensive subjects. Exercise results then suggest that the limitation of the stroke volume is intrinsic to the heart. Lund-Johansen's work ${ }^{7}$ demonstrates that such changes are already present in very mild forms of hypertension.

Our work provides additional information about the nature of the limitation in stroke volume. ${ }^{8}$ Patients with hypertension have faster heart rates, which conceivably could be the reason for the lower calculated stroke volume. This is not the case. After a pharmacologic blockade of the sympathetic and parasympathetic afferents to the heart, the heart rates of both normotensive subjects and patients with borderline hypertension are identical but the stroke volume of the latter remains substantially decreased. ${ }^{8}$

What is the mechanism of this decrease in stroke 
volume? As already discussed, higher afterload is not the likely cause; stroke volume remains decreased even when afterload is substantially diminished through the physiologic vasodilation of exercise. Our studies suggest that a decrease in cardiac compliance is the major reason for the decrease in stroke volume in patients with mild hypertension. The evidence is indirect; cardiac compliance cannot be readily measured. Venous return is not impaired in hypertension. Cardiopulmonary blood volume, an excellent index of venous filling, is not decreased in hypertension. In fact, an increase has been found in the very early phases of borderline hypertension. ${ }^{9,10}$ As hypertension advances, cardiopulmonary blood volume returns to the normal range and stroke volume falls. ${ }^{11}$ These findings suggest that the hearts of hypertensive patients become stiffer (less compliant) and fail to stretch sufficiently in response to normal venous filling. The earliest clinical sign of this altered compliance is the $\mathrm{S}_{4}$ sound in patients with advanced hypertension. Modern echo-Doppler methods have revealed a diastolic filling abnormality during the very early phases of hypertension. ${ }^{12-14}$

Whereas decreased cardiac compliance is the proximate mechanism for the lower stroke volume in hypertension, the increased afterload is its primary cause. Chronic blood pressure elevation causes not only myocardial hypertrophy; a decrease in the cardiac compliance is one of the earliest manifestations of hypertension.

\section{ALTERED CARDIAC AND VASCULAR RESPONSIVENESS}

The autonomic control of the circulation depends on the relationship between the centrally generated tone and the peripheral responsiveness to that tone. Hypertension and aging alter the cardiovascular responsiveness to sympathetic stimulation.

Infusions of the $\beta$-adrenergic agonist isoproterenol cause smaller increases in heart rate and cardiac output in hypertensive patients than in normotensive subjects. ${ }^{8,15} \beta$-Adrenergic-mediated vasodilation is also diminished in hypertension. ${ }^{16}$ Similar decreases in $\beta$-adrenergic responsiveness of the heart ${ }^{17,18}$ and blood vessels have been observed with aging..$^{19} \mathrm{At}$ the biochemical level, aging diminishes $\beta$-adrenergically stimulated generation of adenyl cyclase. ${ }^{20,21}$ With aging, both a decreased number of $\beta$-adrenergic receptors (down-regulation) and an uncoupling between the receptors and their second messenger ${ }^{22}$ seem to be at play.

Increased $\alpha$-adrenergic responsiveness is a welldocumented phenomenon in hypertension. ${ }^{23,}{ }^{24} \mathrm{~A}$ similar age-related enhanced responsiveness of $\alpha$ adrenergic receptors has not been reported. In fact it has been suggested that the pressure response to $\alpha$ adrenergic stimulation is decreased with aging ${ }^{25}$ because of an uncoupling between the receptor and its biochemical mediator similar to that reported for $\beta$ receptors.

The nature of the $\alpha$-adrenergic hyperreactivity in hypertension has not been fully elucidated. There is no doubt that vascular hypertrophy is a major factor, ${ }^{26}$ but a specific hypersensitivity of receptors and calcium-mediated $\alpha$-adrenergic contraction may also play a role. ${ }^{24}$ From a practical standpoint, it is important to understand the amplifying role of arteriolar hypertrophy. This concept has been championed and studied in detail by Folkow. ${ }^{27}$ In the course of hypertension, resistance vessels respond to the increase in work load with a hypertrophy of their medial, muscular layer. The thicker medial layer encroaches on the lumen of the vessel, particularly during vasoconstriction. Vascular resistance is a fourth power function of the vessel's lumen. Even minor increases in wall thickness greatly amplify vascular resistance during vasoconstriction. Higher pressure is required to overcome the increased resistance, which sets the stage for a vicious cycle of worsening hypertension. More pressure begets more hypertrophy, which in turn necessitates further increases in blood pressure. Folkow's postulate explains why hypertension is a progressive disease and why early treatment is mandatory to break the vicious cycle.

Since physical properties of a hypertrophic vessel are the reason for its increased reactivity, such a vessel should be overresponsive to all vasoconstrictive stimuli. In our experiments increased responses to norepinephrine and angiotensin have been observed, ${ }^{26}$ but other investigators have found increased responses only to $\alpha$-adrenergic stimulation. ${ }^{23 .}{ }^{24}$ Therefore it is possible that the enhanced $\alpha$-adrenergic responsiveness in hypertension is a combination of a specific $\alpha$-adrenergic hypersensitivity and a nonspecific enhancement of reactivity caused by vascular hypertrophy. Of importance, the structural component of this enhanced $\alpha$-adrenergic responsiveness in hypertension may in itself be dependent on the prevailing sympathetic tone. It is now accepted that sympathetic stimulation is one of the "trophic" factors independent of the blood pressure that in their own right favor cardiovascular hypertrophy. ${ }^{28}$ The trophic effect of sympathetic stimulation on cardiac myocytes is mediated through $\alpha$-adrenergic receptors. ${ }^{29}$ The sympathetics may also play a role in the development of vascular hypertrophy in hypertension. In our studies, the minimal forearm vascular resistance (a measure of vascular hypertrophy) has been positively correlated with 
plasma norepinephrine levels. ${ }^{30}$ It is also well documented that the sympathetic drive is frequently elevated in hypertension. ${ }^{31}$ This elevation is particularly characteristic of younger patients with mild forms of hypertension. ${ }^{32,33}$

In summary, a vicious cycle leads to a progressive elevation of vascular resistance in the course of hypertension. Vascular hypertrophy enhances $\alpha$ adrenergic responsiveness, and enhanced sympathetic tone may favor further vascular hypertrophy.

\section{METABOLIC ABNORMALITIES}

Treatment of hypertension greatly improves the prognosis in regard to strokes and congestive heart failure but has little effect on the excessive coronary morbidity. ${ }^{34}$ This paradox, that high blood pressure is associated with excessive coronary heart disease but blood pressure lowering does not reduce the coronary morbidity, is frequently explained by the negative effect of diuretics and $\beta$-blocking agents on patients' lipid profiles. The evidence to support this explanation is weak and inferential. Almost entirely forgotten in the dilemma is the fascinating association between hypertension and coronary risk factors. There is no doubt that high blood pressure per se causes atherosclerosis; after coronary bypass surgery, atherosclerosis-free veins that are placed in the high-pressure circuit soon develop atherosclerosis. However, in addition to excess atherosclerosis because of higher pressure, patients with hypertension have several independent risk factors that in their own right are conducive to atherosclerosis. Dyslipidemias and hyperinsulinemia are found in a large proportion of patients with hypertension. ${ }^{35}$, 36 Hyperinsulinemia is an independent risk factor for atherosclerosis. ${ }^{37} \mathrm{~A}$ recent study conducted in Tecumseh, Mich., sheds further light on this relationship. ${ }^{38}$ Middle-aged subjects (average age 31 years) with borderline hypertension had significantly elevated levels of cholesterol, triglycerides, and insulin and an increased insulin/glucose ratio, whereas their lowdensity lipoprotein cholesterol level was significantly decreased. The relationship between the systolic blood pressure and coronary risk factors was also seen in the overall population of normotensive subjects. This relationship, to a large degree, reflected the association between overweight and blood pressure. Overweight, in turn, becomes more of a problem with increasing age. For example, in Tecumseh overweight became a noticeable correlate of the blood pressure only after puberty. ${ }^{38}$

The similarity between the effects of hypertension and aging on plasma lipids and plasma insulin levels is also fascinating. Cholesterol and insulin levels increase with age. Middle-aged patients with hypertension are more likely to show lipid abnormalities and may have the first signs of insulin resistance. The nature of the association of aging and hypertension with hyperinsulinemia is not yet understood, but some useful hints come from the results of treatment of hypertension. Only two categories of drugs are known to actually improve insulin resistance: $\alpha$-adrenergic-blocking agents ${ }^{39}$ and converting enzyme inhibitors. ${ }^{40}$ Both are vasodilators. It is reasonable to speculate that hypertension and aging may decrease the blood supply to a tissue critical for insulin's effect on glucose and that vasodilation may improve it.

\section{CLINICAL IMPLICATIONS}

Treatment of hypertension is entirely empiric. Antihypertensive compounds are developed in chemical laboratories, tested in efficacy trials, and then released for practical use. The primary goal is to lower the blood pressure regardless of the underlying cause of hypertension. Before the advent of the second generation of antihypertensive compounds, all sympatholytic agents caused fluid retention, and vasodilators could not be used without sympatholytic agents to buffer the reflex activation of the sympathetics. Therefore uniform stepped care treatment of hypertension was devised, starting with diuretics, adding sympatholytics, and finally, if needed, vasodilators. Although this approach has yielded impressive results in reducing the morbidity and mortality of hypertension, it is intellectually unsatisfactory. One would like to have some firm system for choosing an antihypertensive compound in a given patient. How can a physician defend the decision to use one drug rather than another similar drug? The proposed solutions usually take into account one of three possible criteria: demographics, pathophysiology, or claim of a specific advantage beyond antihypertensive efficacy. Certain drugs have been recommended for young versus old, black versus white, and overweight versus normal-weight persons. Pathophysiologic classification into volume versus vasoconstriction types of hypertension is an example of a pathophysiologic approach to the choice of antihypertensive agents. An example of a claimed specific advantage of an antihypertensive drug is the recent suggestion that converting enzyme inhibitors offer better protection against renal failure in hypertensive diabetic patients. All these approaches rest on a good rationale, and some of them are supported by actual experimental results. However, the clinical advantage of these recommendations need not be overemphasized. Exceptions to the proposed rules abound. The achievements of stepped care treatment of hyper- 
tension are considerable, and new approaches, while intellectually appealing, should not divert the focus from the need to achieve a normal blood pressure. The primary goal of antihypertensive therapy is still to lower the blood pressure. Only if that goal is achieved can one engage in matching the patient with the specific drug. Consequently, while marshalling pathophysiologic evidence that generally favors the use of vasodilators, particularly $\alpha$-adrenergic vasodilators, in middle-aged patients with hypertension, I do not wish to overstate the case. If an $\alpha$-adrenergic-blocking agent is efficacious, and this is frequently the case, it is well suited for use in middleaged patients with hypertension.

The circulatory effects of hypertension and aging are similar, and the hemodynamics of a middle-aged patient with hypertension are akin to those of an older, normotensive person. Such a patient has elevated vascular resistance at rest, is not able to vasodilate sufficiently during physical exercise, and has a fixed decrease in stroke volume. The sympathetic tone has shifted from a less-responsive heart to more-responsive resistance vessels. This increased dependence of the vascular resistance on the prevailing $\alpha$-adrenergic tone suggests that lowering blood pressure with $\alpha$-adrenergic-blocking agents is an appropriate pathophysiologic intervention in such patients. The growing evidence that sympathetic stimulation through an $\alpha$-adrenergic trophic effect enhances cardiac hypertrophy ${ }^{29}$ further speaks for the use of $\alpha$-adrenergic-blocking agents in middleaged patients. Vascular hypertrophy is an important amplifying mechanism in hypertension. If it is left unattended, the progressive vascular hypertrophy leads to more vascular resistance, increased responsiveness to all vasoconstrictive stimuli, and worsening of the blood pressure. ${ }^{2 ?}$

On a practical level, it is very important to lower the blood pressure in a middle-aged patient with drugs that do not negatively affect exercise performance. Physical exercise is an adjuvant to weight control, improves insulin resistance, and contributes to the patient's feeling of well-being. Unless a middle-aged person makes a conscious effort to exercise regularly, a growing family, increasing business responsibilities, and the loss of former recreational partners are likely to decrease the amount of exercise obtained. Adding antihypertensive agents that cause fatigue or cramps further discourages exercise. Because they do not diminish the exercise capacity, converting enzyme inhibitors, calcium antagonists, and $\alpha$-adrenergic-blocking agents are preferred over $\beta$-blocking agents and diuretics in most middle-aged patients with hypertension.
Because both aging and hypertension are intimately associated with dyslipidemia and insulin resistance, it is very important to determine whether a middle-aged patient with hypertension has these coronary risk factors. Diuretics and $\beta$-blocking agents tend to have a negative effect on plasma lipid levels and should not be used in patients with dyslipidemias. Whereas calcium antagonists and converting enzyme inhibitors are lipid neutral, $\alpha$-adrenergicblocking agents actually cause a modest decrease in plasma lipid levels. ${ }^{41-43} \mathrm{New}$ findings of an improvement in insulin sensitivity in patients treated with converting enzyme inhibitors and with an $\alpha$-adrenergic-blocking agent ${ }^{39,40}$ are promising.

Specific clinical trials are needed to see whether the theoretic advantages of $\alpha$-adrenergic blockers can be translated into a positive clinical effect. In addition, the fact that some antihypertensive drugs improve the underlying metabolic abnormality is of great scientific interest. It may be possible to use $\alpha$ adrenergic-blocking agents, converting enzyme inhibitors, and other vasodilators as research tools to unravel the relationship between hypertension and insulin resistance. If the common denominator is the hemodynamic action of vasodilators, then studies of patterns of regional vasodilation versus the effect on insulin sensitivity may be very useful. Analysis of the few studies in the literature suggests that $\alpha_{1}$-adrenergic-blocking agents may have a more potent effect on insulin resistance than do other compounds. If this finding is replicated in other studies, an additional relationship between the sympathetic overactivity in hypertension and the development of insulin resistance may be postulated.

In conclusion, age- and hypertension-related cardiovascular and metabolic changes provide a good rationale for the use of $\alpha$-adrenergic-blocking agents in middle-aged patients with hypertension. Some of the advantages of $\alpha_{1}$-blockade, such as a positive effect on blood lipid levels and the absence of negative effects on exercise performance, are sufficiently proved and deserve to be incorporated into the therapeutic decision. Other advantages are still speculative. Nevertheless, if an $\alpha$-adrenergic-blocking agent causes no side effects and induces a good blood pressure response, it should be a preferred treatment for a middle-aged subject with hypertension.

\section{REFERENCES}

1. Julius S, Amery A, Whitlock LS, Conway J. Influence of age on the hemodynamic response to exercise. Circulation 1967; 36:222-30.

2. Brandfonbrener M, Landowne M, Shock NW. Changes in cardiac output with age. Circulation 1955;12:557-66.

3. Granath A, Jonsson B, Strandell T. Circulation in healthy old 
men, studied by right heart catheterization at rest and during exercise in supine and sitting position. Acta Med Scand 1964;176:425-46.

4. Rodeheffer RJ, Gerstenblith G, Becker LC, Fleg JL, Weisfeldt ML, Lakatta EG. Exercise cardiac output is maintained with advancing age in healthy human subjects: cardiac dilation and increased stroke volume compensate for a diminished heart rate. Circulation 1984;69:203-13.

5. Conway J, Julius S, Amery A. Effect of blood pressure level on the hemodynamic response to exercise. Hypertension 1968;16: $79-85$.

6. Amery A, Julius S, Whitlock LS, Conway J. Influence of hypertension on the hemodynamic response to exercise. Circulation 1967;36:231-7.

7. Lund-Johansen P. Hemodynamics in early essential hypertension. Acta Med Scand 1987;482(suppl):1-105.

8. Julius S, Randall OS, Esler MD, Kashima T, Ellis CN, Bennett J. Altered cardiac responsiveness and regulation in the normal cardiac output type of borderline hypertension. Circ Res 1975;36-37(suppl I):I-199-I-207.

9. Ellis CN, Julius S. Role of central blood volume in hyperkinetic borderline hypertension. Br Heart J 1973;35:450-5.

10. Safar ME, Weiss YA, London GM, Frackowiak RF, Milliez PL. Cardiopulmonary blood volume in borderline hypertension. Clin Sci Mol Med 1974;47:153-64.

11. Julius $\mathrm{S}$. Transition from high cardiac output to elevated vascular resistance in hypertension. AM HEART J 1988;116:600-6.

12. Fouad FM, Slominski JM, Tarazi RC. Left ventricular diastolic function in hypertension: relation to left ventricular mass and systolic function. J Am Coll Cardiol 1981;3:1500-6.

13. Inouye I, Massie B, Loge D, et al. Abnormal left ventricular filling: an early finding in mild to moderate systemic hypertension. Am J Cardiol 1984;53:120-6.

14. Meloni L, Ruscazio M, Lai L, Mercuro G, Cherchi A. Different patterns of left ventricular filling in arterial hypertension. Eur Heart J 1990;11:302-10.

15. Trimarco B, Volpe M, Ricciardelli B, et al. Studies of the mechanisms underlying impairment of beta-adrenoreceptormediated effects in human hypertension. Hypertension 1983;5:584-90.

16. Bühler FR, Kiowski W, Bolli P, Müller FB, Jones RC. The $\beta$ and $\alpha$-adrenoceptor response adaptation in hypertension development. J Cardiovasc Pharmacol 1987;10(suppl 4):S76S80.

17. Vestal RE, Wood AJJ, Shand DG. Reduced $\beta$-adrenoreceptor sensitivity in the elderly. Clin Pharmacol Ther 1979;26:181-6.

18. Guarnieri T, Filburn CR, Zitnik G, Roth GS, Lakatta EG. Contractile and biochemical correlates of $\beta$-adrenergic stimulation of the aged heart. Am J Physiol 1980;239:H501-H508.

19. van Brummelen P, Bühler FR, Kiowski W, Amann FW. Agerelated decrease in cardiac and peripheral vascular responsiveness to isoprenaline: studies in normal subjects. Clin Sci 1981;60:571-7.

20. Abrass IB, Scarpace PJ. Catalytic unit of adenylate cyclase: reduced activity in aged-human lymphocytes. J Clin Endocrinol Metab 1982;55:1026-8.

21. Scarpace PJ. Decreased $\beta$-adrenergic responsiveness during senescence. Fed Proc 1986;45:51-4.

22. Feldman RD, Limbird LE, Nadeau J, Robertson D, Wood AJJ. Alterations in leukocyte $\beta$-receptor affinity with aging: a potential explanation for altered $\beta$-adrenergic sensitivity in the elderly. N Engl J Med 1984;310:815-9.

23. Amann FW, Bolli P, Kiowski W, Bühler FR. Enhanced alphaadrenoreceptor-mediated vasoconstriction in essential hypertension. Hypertension 1981;3(suppl I):I-119-I-123.

24. Philipp Th, Distler A, Cordes U. Sympathetic nervous system and blood-pressure control in essential hypertension. Lancet 1978;2:959-63.

25. Elliott HL, Sumner DJ, McLean K, Reid JL. Effect of age on the responsiveness of vascular $\alpha$-adrenoceptors in man. J Cardiovasc Pharmacol 1982;4:388-92.

26. Egan B, Panis R, Hinderliter A, Schork N, Julius S. Mechanism of increased alpha adrenergic vasoconstriction in human essential hypertension. J Clin Invest 1987;80:812-7.

27. Folkow B. Physiologic aspects of primary hypertension. Physiol Rev 1982;62:347-503.

28. Hart MN, Heistad DD, Brody MJ. Effect of chronic hypertension and sympathetic denervation on wall/lumen ratio of cerebral vessels. Hypertension 1980;2:419-28.

29. Simpson P. Norepinephrine-stimulated hypertrophy of cultured rat myocardial cells is an alpha $a_{1}$ adrenergic response. $J$ Clin Invest 1983;72:732-8.

30. Egan B, Julius S. Vascular hypertrophy in borderline hypertension: relationship to blood pressure and sympathetic drive. Clin Exp Hypertens [A] 1985;A7:243-55.

31. Julius $\mathrm{S}$. Role of the sympathetic nervous system in the pathophysiology of cardiovascular disease. AM HEART J 1987;114:232-4.

32. Esler M, Jennings G, Biviano B, Lambert G, Hasking G. Mechanism of elevated plasma noradrenaline in the course of essential hypertension. J Cardiovasc Pharmacol 1986;8(suppl 5):S39-S43.

33. Goldstein DS. Plasma norepinephrine in essential hypertension: a study of the studies. Hypertension 1981;3:48-52.

34. Veterans Administration Cooperative Study Group on Antihypertensive Agents. Effects of treatment on morbidity in hypertension: II. Results in patients with diastolic blood pressure averaging 90 through $114 \mathrm{~mm} \mathrm{Hg.} \mathrm{JAMA} \mathrm{1970;213:1143-52.}$

35. Williams RR, Hunt SC, Hopkins PN, et al. Familial dyslipidemic hypertension: evidence from 58 Utah families for a syndrome present in approximately $12 \%$ of patients with essential hypertension. JAMA 1988;259:3579-86.

36. Ferrannini E, Buzzigoli G, Bonadonna $R$, et al. Insulin resistance in essential hypertension. N Engl J Med 1987:317:350-7.

37. Dulcimetiere P, Eschwege E, Papoz L, Richard JL, Claude JR, Rosselin G. Relationship of plasma insulin levels to the incidence of myocardial infarction and coronary heart disease mortality in a middle-aged population. Diabetologia 1980;19: 205-10.

38. Julius S, Jamerson K, Mejia A, Krause L, Schork N, Jones K. The association of borderline hypertension with target organ changes and higher coronary risk: Tecumseh blood pressure study. JAMA 1990);264:354-8.

39. Pollare T, Lithell H, Selinus I, Berne C. Application of prazosin is associated with an increase of insulin sensitivity in obese patients with hypertension. Diabetologia 1988;31:41520.

40. Pollare T, Iithell H, Rerne C. A comparison of the effects of hydrochlorothiazide and captopril on glucose and lipid metabolism in patients with hypertension. $N$ Engl $J$ Med 1989;321:868-73.

41. Frick MH, Cox DA, Himanen P, et al. Serum lipid changes in a one-year, multicenter, double-blind comparison of doxazosin and atenolol for mild to moderate essential hypertension. Am J Cardiol 1987;59:61G-7G.

42. Talseth T, Westlie L, Daae L, Vatle S. Comparison of the effects of doxazosin and atenolol on blood pressure and blood lipids: a one-year, double-blind study in 228 hypertensive patients. AM HEART J 1988;116:1790-6.

43. Ames RP. The effects of antihypertensive drugs on serum lipids and lipoproteins: II. Non-diuretic drugs. Drugs 1986;32: 335-57. 